\title{
Development of friction-induced triboluminescent sensor for load monitoring
}

Journal of Intelligent Material Systems and Structures 2018, Vol. 29(5) 883-895 (c) The Author(s) 2017 Reprints and permissions: sagepub.co.uk/journalsPermissions.nav DOI: 10.II77//045389XI7721049 journals.sagepub.com/home/jim (SAGE

\author{
Md Abu S Shohag', Zhengqian Jiang', Emily C Hammel', \\ Lucas Braga Carani ${ }^{2}$, David O Olawale ${ }^{3}$, Tarik J Dickens', \\ Hui Wang' and Okenwa I Okoli'
}

\begin{abstract}
Real-time load monitoring of critical civil and mechanical structures especially dynamic structures such as wind turbine blades is imperative for longer service life. This article proposed a novel sensor system based on the proprietary in situ triboluminescent optical fiber (ITOF) sensor for dynamic load monitoring. The new ITOF sensor patch consists of an ITOF sensor network with micro-exciters integrated within a polymer matrix. The sensor patch was subjected to repeated flexural loading and produced triboluminescent emissions due to the friction between micro-exciters and ITOF sensors corresponding to each loading cycle. The friction-induced triboluminescent intensity directly depends on the loading rate, the coefficient of friction, and the applied load on patch. In general, the triboluminescent intensity increases exponentially with an increase in load. Additionally, the sensor patches comprising the coarser micro-exciters exhibited better results. Similarly, better results were achieved at higher loading rates although a threshold loading rate is required to excite the triboluminescent crystals for this sample configuration. The proposed new sensor has the ability to monitor dynamic continuous applied loads.
\end{abstract}

\section{Keywords}

Triboluminescence, friction, sensor, load monitoring, flexural loading, sensor patch

\section{Introduction}

Triboluminescent (TL) materials emit light when they are scratched, stressed, or fractured (Bergeron et al., 2006; Sweeting, 2001), and this fracture-induced light emission phenomenon is called triboluminescence (Sage et al., 1999). The term consists of two parts: the first is the Greek word tribein that means "to rub," while the second is the Latin prefix lumen, meaning "light" (Womack et al., 2003). Hence, this term essentially means light from friction (Goedeke et al., 2003).

There are thousands of different TL materials including both organic and inorganic materials. Among all crystals, approximately $50 \%$ of inorganic crystals and $30 \%$ of organic crystals exhibit TL behavior (Walton, 1977). Xu et al. (1999b) showed a comparison of the TL intensity of various inorganic materials under identical mechanical stress conditions. The comparison clearly indicates that the manganese-doped zinc sulfide (ZnS:Mn) has the highest intensity TL emissions, which allows for more light emitted with fewer crystals than other TL crystals. The highest intensity is found at manganese doping level of about 5 at $\%$ of $\mathrm{ZnS}(\mathrm{Xu}$ et al., 1999b). On the other hand, the organic europium dibenzoylmethide triethylammonium (EuD4TEA) doped with dimethyl methylphosphonate (DMMP; LM-194) has the largest tested TL yield among all the tested materials (Hollerman et al., 2012). However, it appears to be more easily damaged than inorganic ZnS:Mn (Olawale et al., 2016a). Thus, it is concluded that ZnS:Mn has the most potential for TL-based sensor systems and it was used as the TL material for this research.

Many studies have been performed on various TL crystals to correlate the TL emission and the applied

\footnotetext{
'High-Performance Materials Institute, Florida A\&M University-Florida State University College of Engineering, Tallahassee, FL, USA

${ }^{2}$ Sao Paulo State University (UNESP), Sao Paulo, Brazil

${ }^{3}$ R.B. Annis School of Engineering, The Shaheen College of Arts and Sciences, University of Indianapolis, Indianapolis, IN, USA
}

\section{Corresponding author:}

Okenwa I Okoli, High-Performance Materials Institute, Florida A\&M

University-Florida State University College of Engineering, 2525

Pottsdamer Street, Tallahassee, FL 32310, USA.

Email: okoli@eng.fsu.edu 
loads (Chandra and Chandra, 2011; Jia et al., 2006; Xu et al., 1999a). In most of those studies, the load is applied directly normal to the TL crystals. For instance, $\mathrm{Xu}$ et al. (1999b) reported that the TL intensity increased sharply when friction was applied normal to the $\mathrm{ZnS}: \mathrm{Mn}$ thin film. The study also showed that the TL emission intensity increased with the applied loads. On the other hand, Leelachao et al. (2016) showed that the friction-induced TL emission of $\mathrm{ZnS}: \mathrm{Mn}$ dispersed in polymer matrix is proportional to applied normal force. Moreover, such linear relationship between TL intensity and applied load has been reported in numerous studies relating to friction-induced triboluminescence (Xu et al., 1999a; Zhang et al., 2013). In addition, an empirical relationship was proposed by Leelachao et al. (2016) where the TL emission intensity was expressed in the form of an exponential function of a square of the applied stress. Several other studies on the relationship of the TL emission and stress can be found in other works (Chandra and Chandra, 2011; Jia et al., 2006; Someya et al., 2013).

A lot of research has been done on TL-based sensors as damage and impact monitoring systems in engineering systems such as civil and aerospace structures. To the authors' knowledge, no research has been published on TL sensors that could monitor real-time loads on structures such as wind blades. Conventional strain gauges located in the root section of the blades can be used for load measurement through correlation of strain. Some limitations of the sensor are as follows: (1) it needs calibration periodically, (2) it is influenced by temperature variations, and (3) it is sensitive to lightning and electromagnetic fields (Ozbek et al., 2009). Currently, fiber Bragg grating (FBG) sensors, the most common fiber optic sensors, have been used for monitoring various types of civil infrastructure (Chan et al., 2006; Ho et al., 2013; Leng et al., 2006; Majumder et al., 2008; Moyo et al., 2005). As a result, FBGs are promising candidates for wind blade load monitoring due to their many advantageous characteristics (Schroeder et al., 2006). The challenging part of implementing these fiber optic sensors is the need for expensive and bulky interrogation equipment, which may prevent its widespread field application in wind turbine blade monitoring ( $\mathrm{Li}$ et al., 2015). It is therefore imperative to develop a new cost-effective sensor system that can accurately measure loads on blades in real-time.

The focus of this study is to develop a new sensor system for load monitoring which is based on the proprietary in situ triboluminescent optical fiber (ITOF) sensor (Olawale, 2013; Olawale et al., 2011, 2014, 2016b). Figure 1 is a schematic diagram of the ITOF sensor. The ITOF sensor has the capability of detecting in situ damage in cementitious composites (Olawale et al., 2014, 2016b) or monitoring real-time damage in the adhesive bond of composite structures (Shohag et al., 2016b). The new sensor network patch, ITOFPress consists of an ITOF sensor network with micro-exciters integrated within a polymer matrix. The proposed sensor network could provide real-time quasi-distributed load information on structures such as composite wind turbine blades. This sensor is particularly suitable for large and remotely located composite structures such as the wind blades of utility scale wind turbines. Unlike many other sensors, the ITOFPress sensor does not require external power source (battery) for load sensing and transmission. This is critical

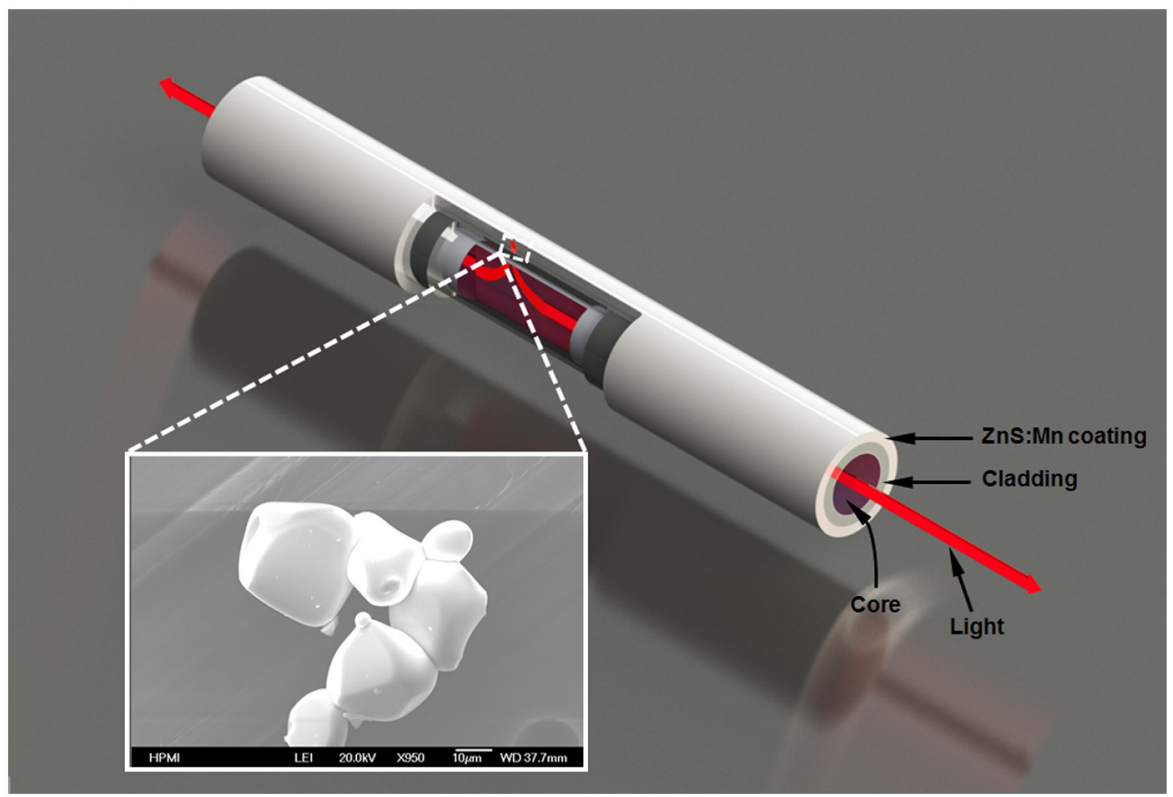

Figure I. Illustration of the ITOF sensor. The inset shows the ZnS:Mn crystals. 
because of the associated high cost of maintenance and system downtime required for replacing batterypowered sensors. In addition, it is expected that the cost of the sensor would be comparatively inexpensive due to the lower fabrication cost and noncomplex data acquisition systems.

Mostly in the aforementioned studies, the applied load is static, whereas in this research the applied load is dynamic. The load is not directly applied to the $\mathrm{ZnS}: \mathrm{Mn}$ crystals, but rather through the polyurethane matrix material. The main objective of this article is to report preliminary findings on the new ITOFPress sensor, a new TL-based sensor system for dynamic continuous load monitoring of critical civil and mechanical structures. Additionally, a correlation between TL intensity and loads is suggested based on the results. The article discusses the effects of some crucial design factors such as matrix materials, coarseness of micro-exciters, and micro-exciters' host materials on sensor performance.

\section{Experimentation}

\section{$T L$ sensor fabrication}

A polymer optical fiber (POF) of $1 \mathrm{~mm}$ diameter (Mitsubishi Rayon Company, Japan) was continuously coated with the TL composite film by dip-coating process. The TL composite film consists of $\mathrm{ZnS}: \mathrm{Mn}$ (GL25/N-U1; Phosphor Technology, UK) and a ultraviolet (UV)-cured acrylated urethane system (Dymax Corporation, USA). The particle size of $\mathrm{ZnS}: \mathrm{Mn}$ ranges 5-20 $\mu \mathrm{m}$. TL composite films were fabricated with $50 \%$ by weight of $\mathrm{ZnS}: \mathrm{Mn}$ content. The $\mathrm{ZnS}: \mathrm{Mn}$ crystals were thoroughly mixed in the resin by a Thinky mixer (ARE-310; Thinky Corporation, USA). The Thinky mixer generates planetary centrifugal forces to mix the solution properly. The resulting TL composite film was then deposited on the POF by drawing the POF through a bath containing the TL composite film. The coated POF was cured by passing through a UV lamp for about $20 \mathrm{~s}$.

\section{Material selection and sample fabrication}

The ITOF sensor was strategically placed in a threedimensional (3D) printed mold and allowed to extend beyond the boundary of the mold. The extended length of the ITOF sensor carries the signal from the sensor network patch as described in section "Mechanical tests." A thin film of mold release agent (Pol-Ease 2500; Polytek Development Corp., USA) was sprayed inside the mold to ensure easy part removal. A wide strip of micro-exciters was inserted into the mold and steel pins were used to ensure good contact between the micro-exciters (abrasive particles) and the ITOF sensor. Another purpose of these pins is to hold the sensors in their desired location. The sensor density in a patch increases with an increase in the number of contact points. The number of contact points is determined by the number of times the ITOF within a sensor patch is in close proximity to micro-exciters. It was shown in the previous work Shohag et al. (2016a) that the ITOF sensor patch performed better when the number of contact points was increased to six. The sensor network patch in this experiment was designed to have six contact points. The diameter of the ITOF sensor is around $1.4 \mathrm{~mm}$. The wide strip of micro-exciters is used to increase the contact area between micro-exciters and ITOF sensors. A 456-mm-long ITOF sensor is placed inside the patch matrix, of which $300 \mathrm{~mm}$ is in contact with the micro-exciters. Therefore, the ratio of sensor length covered by the micro-exciters is 0.6579 , that is, $65.79 \%$ of the total ITOF sensor inside the patch matrix. Two different types of polyurethane were used for this experiment as shown in Table 1. In the rest of the article, Poly 75-75 and SpeedSet 401 will be referred to as "Material A" and "Material B," respectively.

The two-part polyurethane was properly mixed and poured into the mold as the ITOFPress matrix material to bind the sensors and micro-exciters together. The ITOFPress was demolded after complete curing (24 h) of the polyurethane resin. The ITOF sensor outside the ITOFPress matrix material was coated with a black tape to insulate the sensor from ambient light. Figure 2 is a picture of ITOFPress.

\section{Mechanical tests}

A setup for the three-point bending tests was developed by fabricating fixtures to hold and apply loads on the samples as shown in Figure 3. The model of the load cell utilized in this experiment is SSM-DAK-5000 N. The rated output (sensitivity) of this load cell is $3 \mathrm{mV} / \mathrm{V}$ and the maximum excitation voltage is $15 \mathrm{~V}$. Preliminary work was done to find out a suitable loading rate that would result in TL signal emission. After several trials $(30,60$, and $120 \mathrm{~mm} / \mathrm{min})$, a loading rate of $120 \mathrm{~mm} / \mathrm{min}$ was selected for this experiment

Table I. Different matrix materials for ITOFPress fabrication.

\begin{tabular}{llll}
\hline Matrix materials & & Supplier & Shore hardness \\
\hline Material A & Poly 75-75 & Polytek Development Corp. & A75 \\
Material B & SpeedSet 40I & Industrial Polymers Corp. & D35-45 \\
\hline
\end{tabular}




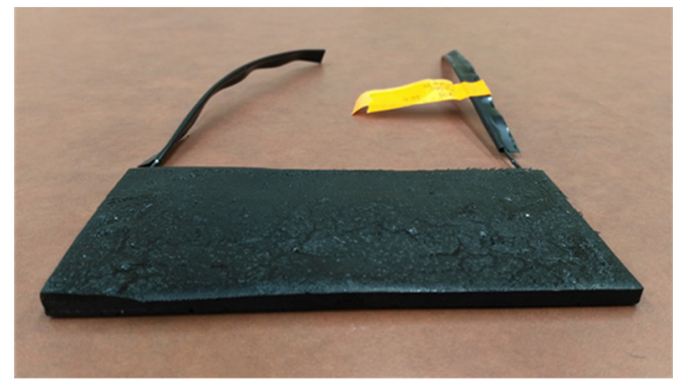

Figure 2. Image of an ITOFPress.

because at the lower loading rates (30 and $60 \mathrm{~mm} / \mathrm{min}$ ) no noticeable TL signal was observed. This suggests a threshold loading rate of $120 \mathrm{~mm} / \mathrm{min}$. The support span of the three-point bending test was $35 \mathrm{~mm}$. A Hamamatsu H10721 series photomultiplier tube (PMT; Hamamatsu, Japan) was connected to the ITOF sensors to monitor the excitation emissions during loading. A data acquisition device from National Instruments (NI USB-6210; USA) was connected to the PMT. A MATLAB program developed in-house converted the
PMT's output voltage into the TL intensity (in arbitrary units).

\section{Microscopy}

A BX40F4 microscope from Olympus Optical Co. Ltd (Japan) was used to perform morphology analysis on the samples and micro-exciters. Four specimens among those used for the optical microscopy were selected for a morphological characterization with scanning electron microscopy (SEM). The microscope used was a JEOL 7401 field emission SEM (JEOL, USA).

\section{Results and discussion}

\section{Performance of ITOFPress under repeated flexural loading}

The typical TL emissions from an ITOFPress during the three-point bending tests are shown in Figure 4(a). The sensors were subject to four loading cycles. Each cycle has two parts called loading half cycle and

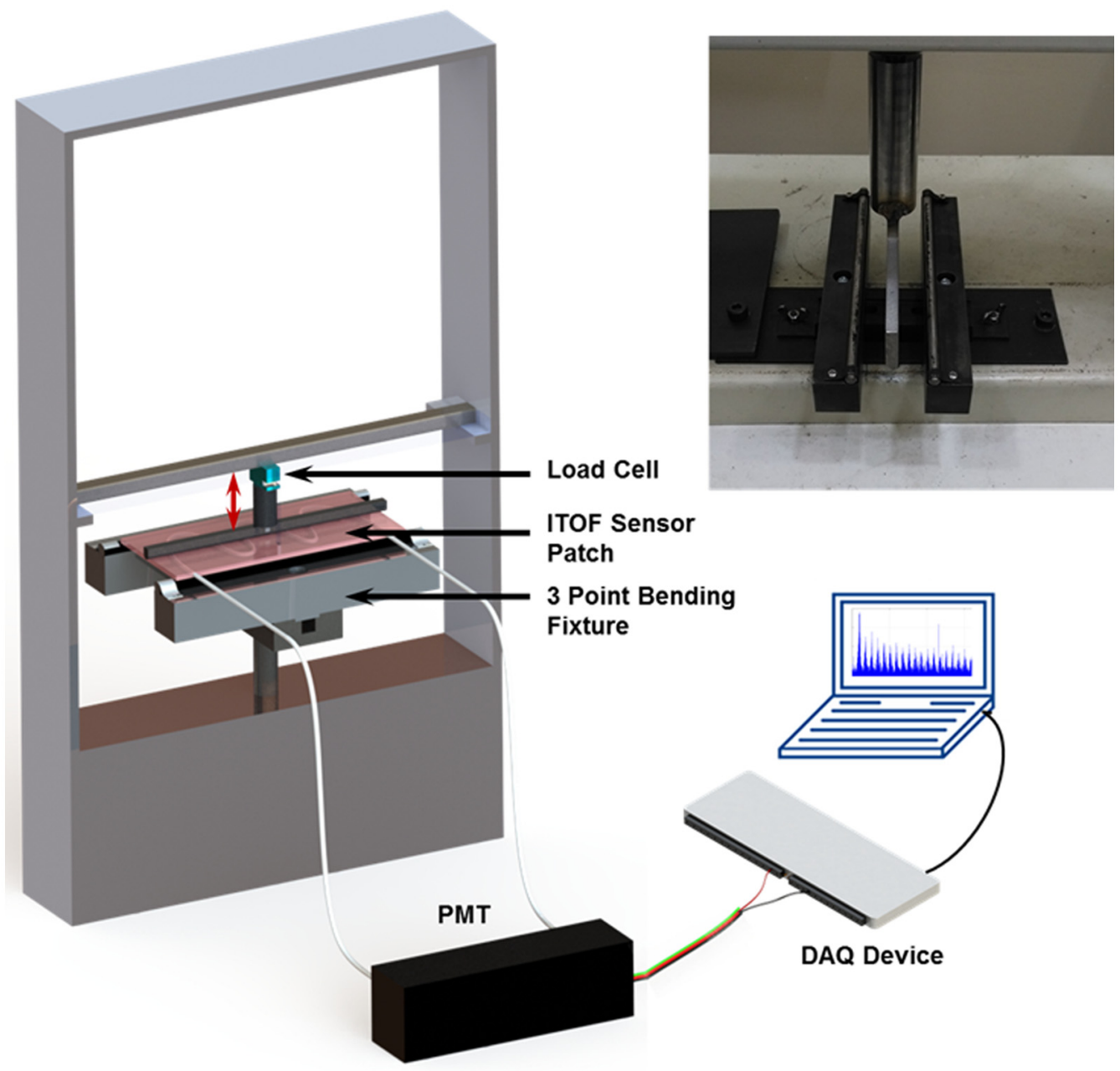

Figure 3. Diagram shows the three-point bending test's setup and the inset is the three-point bending fixture. 


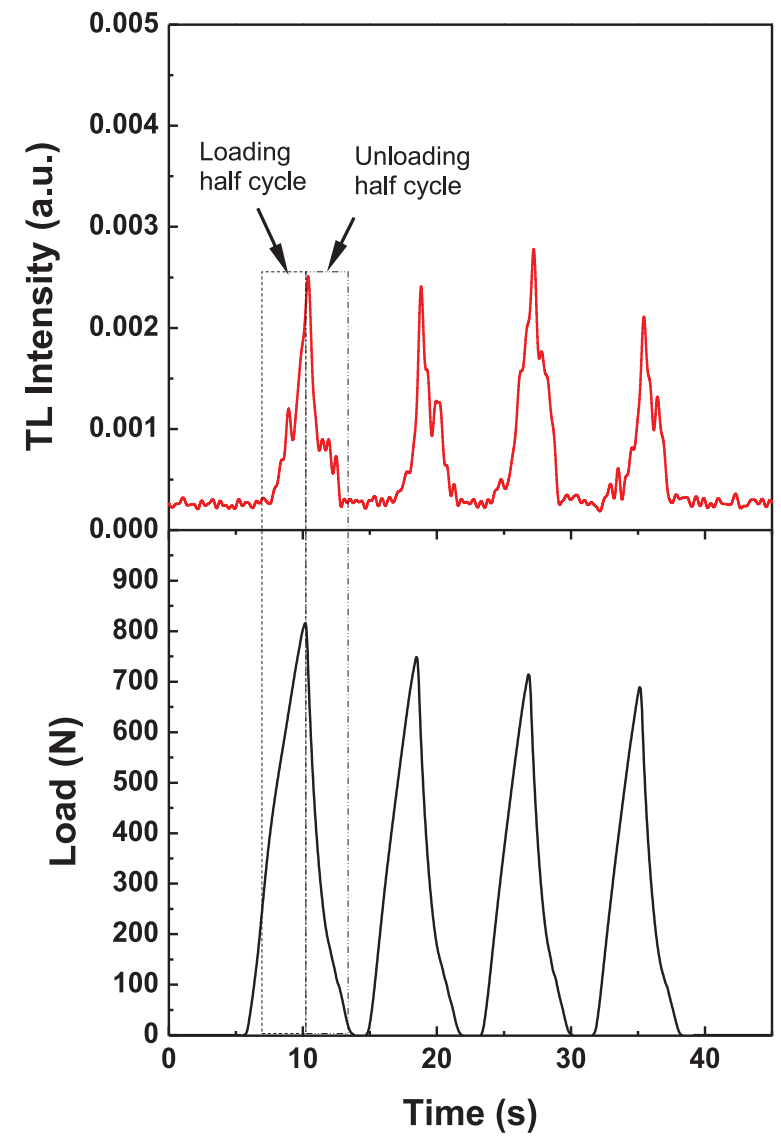

(a)

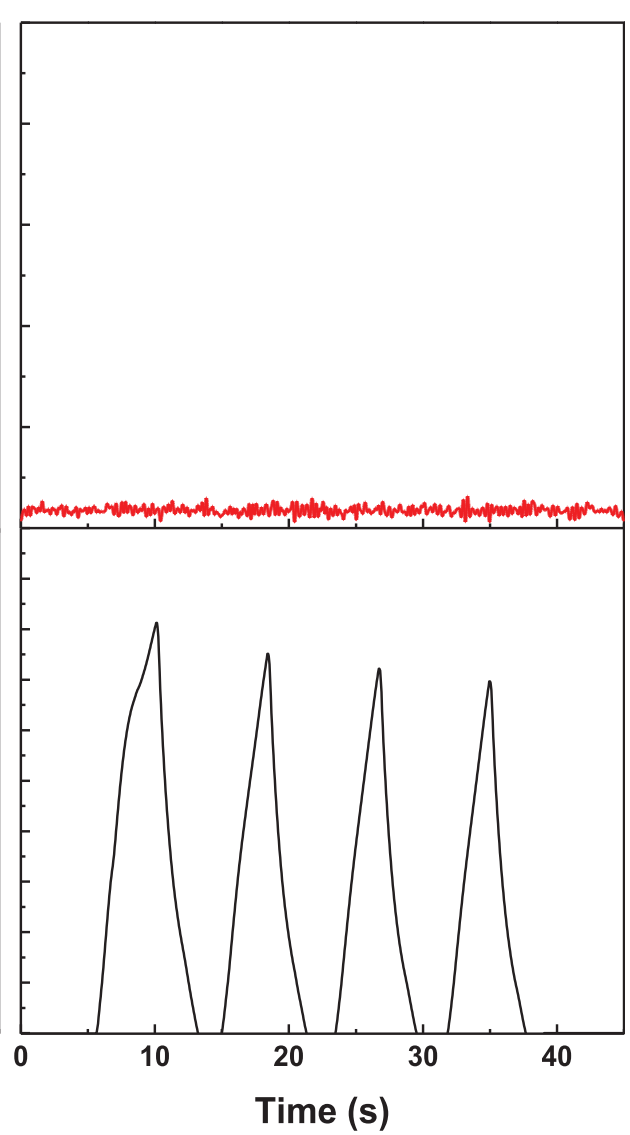

(b)

Figure 4. TL emission due to the repeated loading with respect to time: (a) ITOFPress having micro-exciters and (b) signal output from ITOFPress having no TL crystals on optical fiber due to repeated loads.

unloading half cycle (Figure 4(a)). The ITOFPress produced four TL emissions corresponding to each loading cycle as shown in Figure 4(a). The maximum TL emission was observed at the peak load for each cycle. On the other hand, for the same amount of deformation $(9 \mathrm{~mm})$, Figure $4(\mathrm{~b})$ shows the signal output from a sensor patch that has no TL crystal coating on optical fiber. It displays no signal, only a small amount of noise. Therefore, Figure 4(a) clearly displays the TL emissions from the ITOF sensor patch.

TL intensity $\left(I_{T L}\right)$ is a function of loading rate, coefficient of friction, and applied load on sample as in equation (1)

$$
I_{T L}=f(\dot{F}, \mu, F)
$$

Here, the loading rate $(\dot{F})$ is defined as how fast the load is applied on the sensor network patch and $\mu$ is the coefficient of friction of the micro-exciters. The higher the loading rate, the higher the TL intensity.
Effect of matrix material on sensor performance. Two different grades of polyurethane were used for this analysis, and the corresponding results are shown in Figure 5. Some of the requirements of the matrix material are as follows:

(a) Matrix material should be semi-flexible so that it can bend while loading and allow for little movement of micro-exciters.

(b) Matrix material should have enough stiffness to effectively transfer the load.

ITOF sensor patch with Material A shows more consistent results for each cycle, but the TL intensity is comparatively low (Figure 5). Material A is a semiflexible material. There is a level of material property degradation due to the stress relaxation. With Material $\mathrm{B}$ as the matrix material, TL intensity was markedly enhanced compared to Material A. The ITOFPress having Material B produced high TL intensity levels but inconsistent peak TL signal (Figure 5). As the material has higher stiffness, it could transfer load effectively 


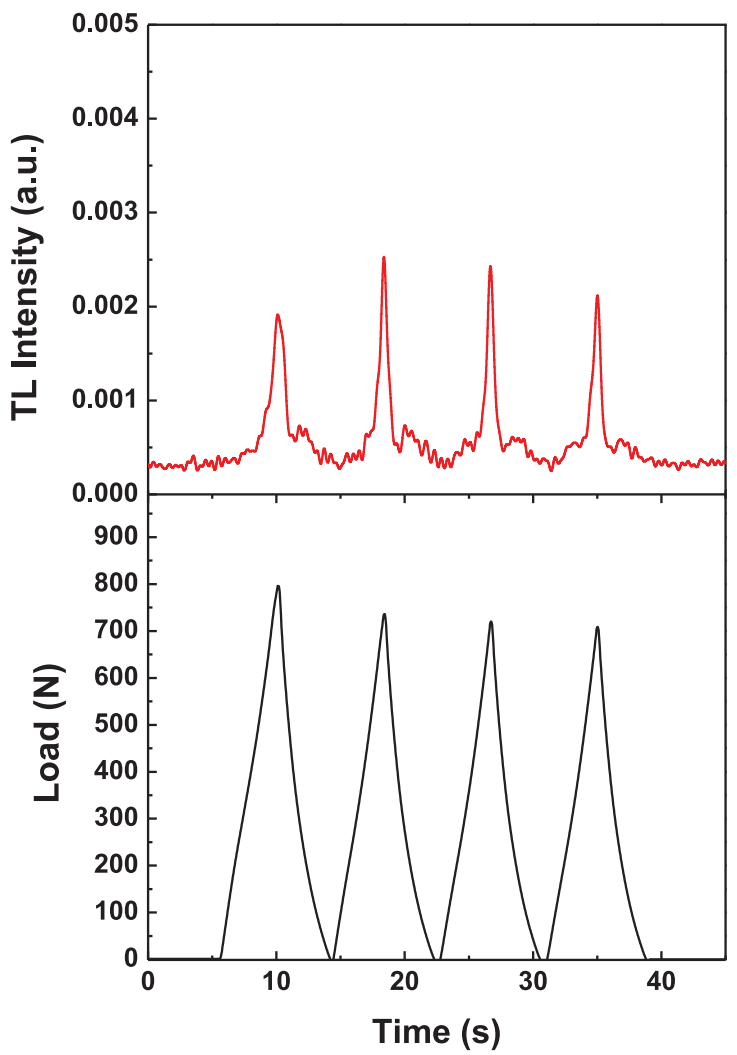

(a)

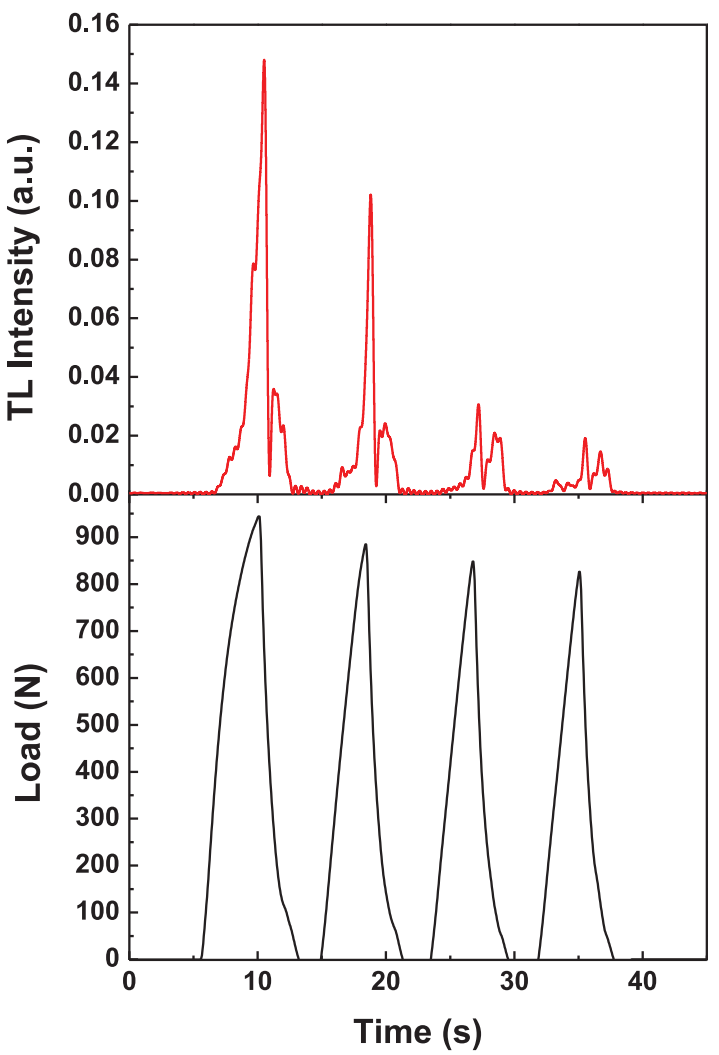

(b)

Figure 5. TL intensity (arbitrary units) due to the repeated loads for the matrix material: (a) Material A and (b) Material B.

to the ITOF sensors for enhanced excitation. The TL emission from these ITOFPress is quite unstable and shows degradation trends. These stiffer ITOFPress are brittle in nature. As such, fractures or cracks occur after several cycles of repeated loading. To generate a consistent and high TL emission, a new patch matrix material needs to be found which has the stiffness in between Material A and Material B and performs excellently under repeated loading.

Effect of micro-exciters' grit size on sensor performance. To determine the effect of the coarseness of micro-exciters on the ITOF sensor patch's performance, two microexciter grit sizes (CAMI scale), namely, 36 and 80, were investigated.

The grit size in microns can be calculated from equation (2)

$$
z=11764.71 n^{-0.93589}
$$

where $z$ is the grit size in microns and $n$ is the grit size in numbers.

The grit size of the micro-exciters in microns is recorded from optical microscopic images, and a comparison between theoretical and experimental grit size

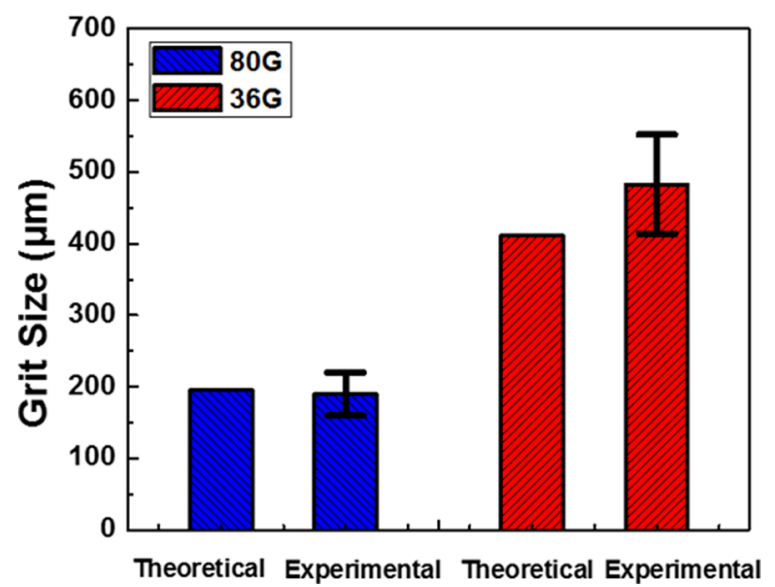

Figure 6. Comparison of theoretical and experimental grit sizes of micro-exciters.

is shown in Figure 6. The coarsest micro-exciters have large particles and high coefficient of friction.

The sensor network patches comprising microexciters with coarser grit (grit size 36) exhibited better results. The distinct and consistent TL emissions for each loading cycle are defined as a "better result" than others. Figure 7 shows the performance comparison between two different sensor network patches 


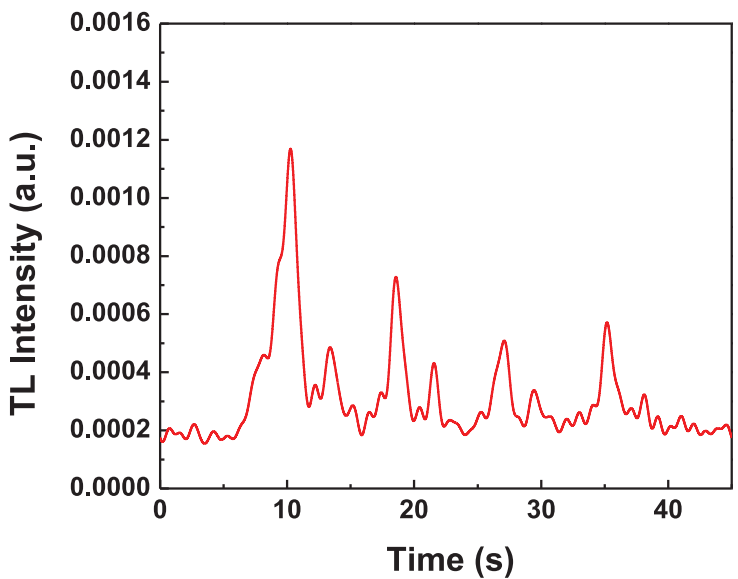

(a)

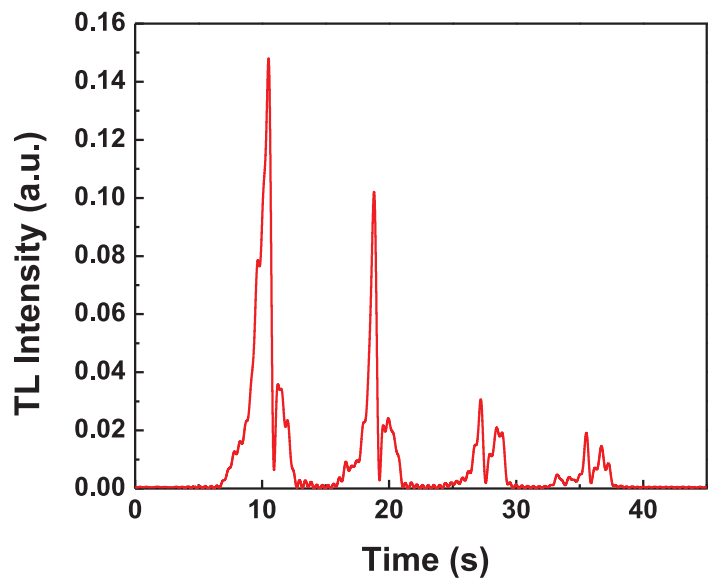

(b)

Figure 7. TL intensity (arbitrary units) due to the repeated loads for the coarseness of the micro-exciters: (a) 80 grit size and (b) 36 grit size. Material $B$ is the matrix material for both the samples.

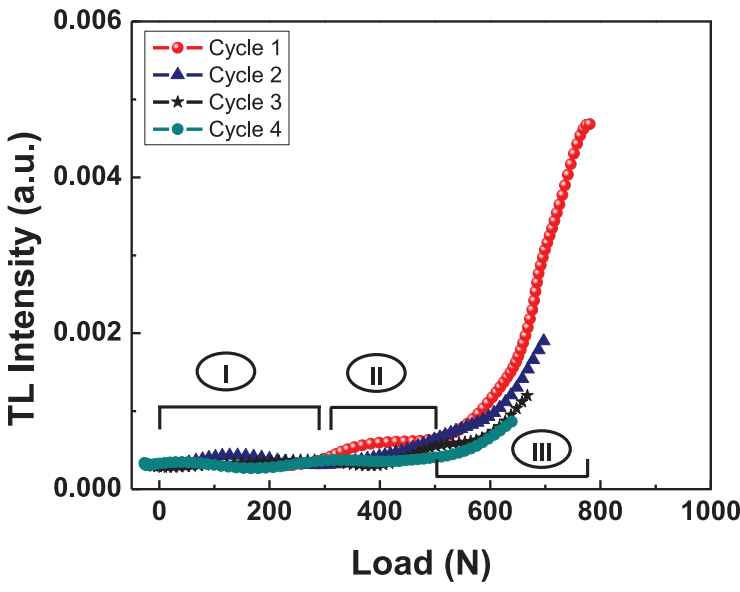

(a)

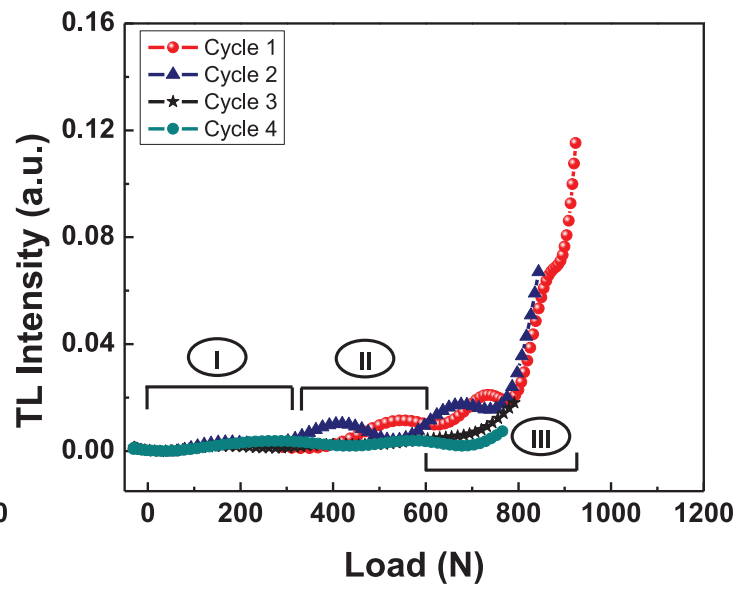

(b)

Figure 8. TL intensity (arbitrary units) of the loading half cycles for the matrix material: (a) Material A and (b) Material B.

comprising grit sizes of 80 and 36 . The sensor network patch comprising 36 grit micro-exciters shows comparatively higher TL emissions than 80 grit size (Figure 7).

\section{Correlation of TL emission intensity with loads}

A correlation between TL intensity and applied load is required for sensor calibration. To understand the relationship between TL intensity and applied load, the signal data in Figure 5 are replotted using load in $x$-axis and TL intensity in $y$-axis. The separate plots for several loading and unloading half cycles are shown in Figures 8 and 9, respectively. Figure 8(a) and (b) shows the TL emissions from the sensor during the loading half cycles for the matrix material "Material A" and "Material B," respectively. In both cases, the TL intensity increases with increased loads. To gain more insights into the TL emission from the sensor patch, the TL intensity versus load curve are split into three regions. In region I (Figure 8(a) and (b)), with the increase in load, the TL intensity increase is insignificant for the first $30 \%$ of the peak loads (approximately 780 and $925 \mathrm{~N}$ for Material A and Material B, respectively, for the first cycle). Under the current loading conditions, the loading force with the range from 0 to $250 \mathrm{~N}$ for Material $\mathrm{A}$ and 0 to $300 \mathrm{~N}$ for Material $\mathrm{B}$ cannot generate enough energy for the TL emissions, that is, the true signal cannot exceed the system errors $(\varepsilon)$. The sensor signal $\left(I_{T L}\right)$ is a combination of true signal $(x)$ and system error $(\varepsilon)$ as shown in equation (3). The true signal is the TL emission only due to the applied force for a fixed loading rate 


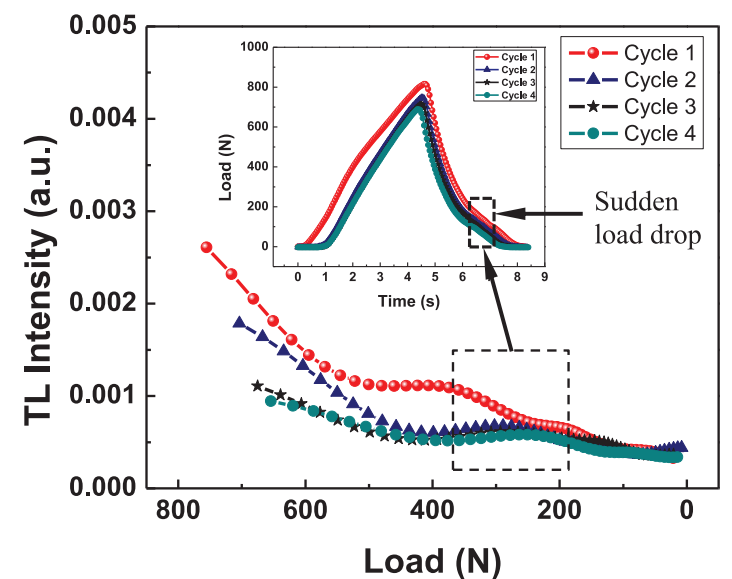

(a)

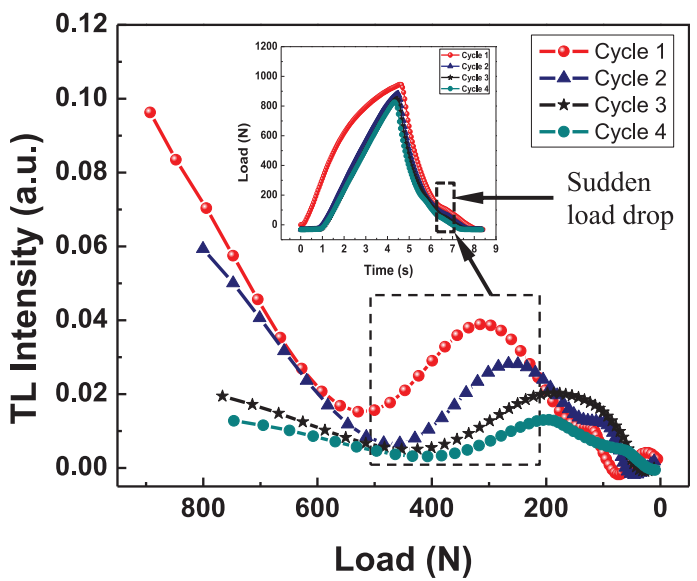

(b)

Figure 9. TL intensity (arbitrary units) of the unloading half cycles for the matrix material: (a) Material A and (b) Material B. The insets show the repeated applied loads on sensor patch with time.

$$
\text { Sensor signal }\left(I_{T L}\right)=\operatorname{True} \operatorname{signal}(x)+\operatorname{System} \operatorname{error}(\varepsilon)
$$

For these sensor configurations, the load sensitivity threshold is therefore around $30 \%$ of the peak load. This threshold value will need to be decreased further to a minimum threshold value for better sensor performance. In region II, the TL emission curve has an upward trend along with a small rate of variation. This kind of instability and uncertainty arises from the friction-based mechanism. There exists some randomness in the friction of the TL sensor which can be considered as the system error ( $(\varepsilon)$ (equation (3)). Additionally, in region II, the true signal generated with the load (ranges from 250 to $500 \mathrm{~N}$ for Material A and 300 to $625 \mathrm{~N}$ for Material B) is still not large enough to overcome the system error. In region III, the true signal is much higher than the system error. As such, TL intensity increases exponentially with the increase in loads in region III starting from approximately 500 and $600 \mathrm{~N}$ for Material A and Material B, respectively.

Similar types of plots were drawn for the unloading half cycles for the matrix material "Material A" and "Material B" as shown in Figure 9. In general, the TL intensity decreases with decreasing loads. Basically, during the unloading half cycle, the load was released gradually over time. In this phase, the TL intensity depends on the material's strain recovery rate. It is expected that the higher the strain recovery rate of the material, the better the TL emission as well as signal consistency.

The inconsistent signal in the region (black dotted box) shown in Figure 9 occurred due to the sudden decrease in load. The non-uniform strain recovery rate of the matrix material could be the reason for signal inconsistency. The material permanently deformed during the loading, thus the load decreased gradually during each cycle and so does the TL intensity. After the test, a fracture was observed on the opposite side of the loaded side of the patch (Material B) which could be the potential cause of the signal inconsistency. In contrast, no fracture was observed in patches with matrix material "Material A." From Figure 10, it is clear that the patch material exhibits stress relaxation over the cycles and carries less amount of load. The unloading paths depict a large hysteresis loop with residual strain that agrees with other research works (Qi and Boyce, 2005; Yi et al., 2006). From the first cycle to second cycle, the decrease in load is quite high compared to the rest of the cycles due to the permanent significant deformation of the composite patch (Figure 10). The cause of this permanent deformation is the brittle nature of the micro-exciters' host material (a thick paper) along with the stress relaxation of polyurethane. Other host materials deserve further study to eliminate the load inconsistency over cycles.

The 3D plots of the TL emissions from an ITOF sensor patch with loads and loading cycles are shown in Figure 11. The TL intensity has a more drastic decrease with increasing number of loading cycles. It is expected that the signal from the sensor stabilizes after several cycles as well as loads for a particular amount of deformation.

For this sensor configuration, an empirical relationship between TL intensity and load is suggested as

$$
I_{T L}=A \exp \left(\frac{F}{B}\right)+C_{0}
$$

where $F$ is the applied load on the sensor patch. In addition, $A, B$, and $C_{0}$ are constants for a particular loading rate and they are a function of loading rate as well 


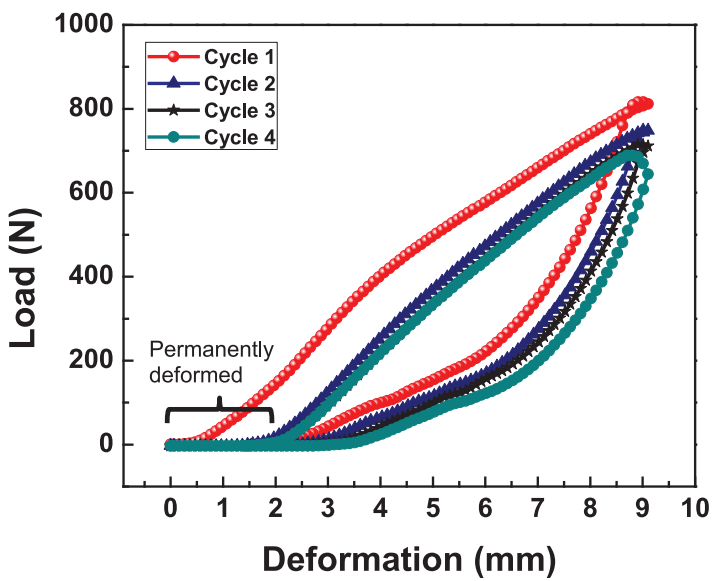

(a)

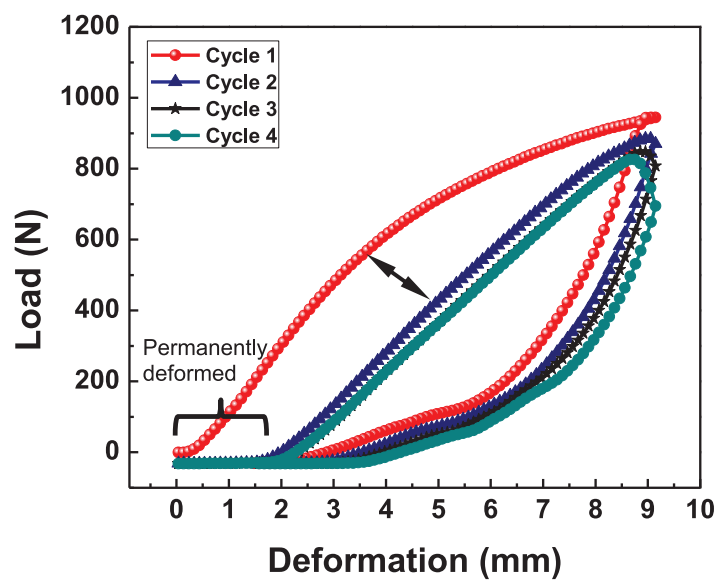

(b)

Figure 10. Repeated applied loads on sensor patch with deformation for the matrix material: (a) Material A and (b) Material B.

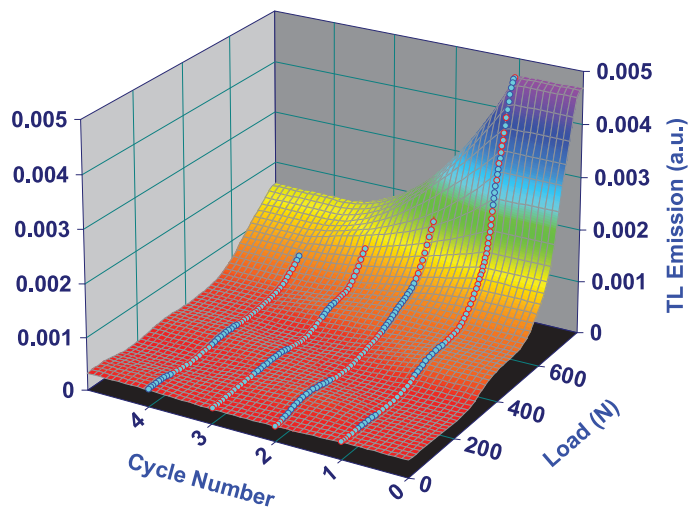

(a)

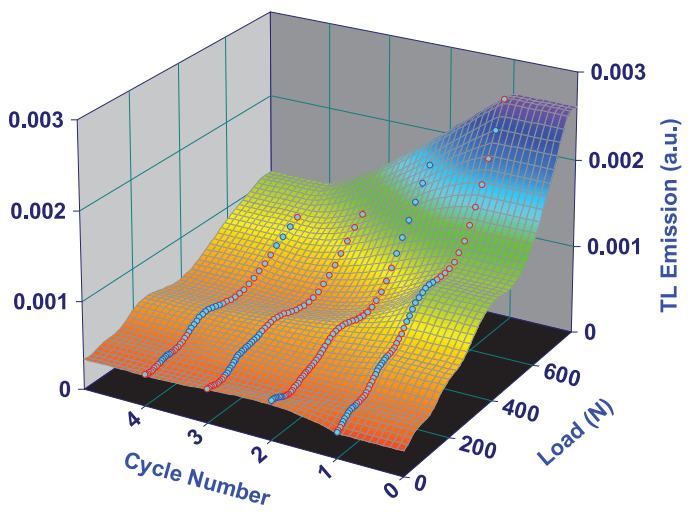

(b)

Figure II. The 3D plots of the TL emissions from ITOF sensor patch with loading cycles and loads: (a) loading half cycle and (b) unloading half cycle.

$$
\begin{aligned}
A & =f(\dot{F}) \\
B & =f(\dot{F}) \\
C_{0} & =f(\dot{F})
\end{aligned}
$$

The limitation of the above empirical equation is that it applies to the single loading investigated in this study for a particular sensor configuration.

\section{Morphological characterization}

Optical microscopy. Four specimens were taken from each sample for optical microscopy. The optical microscopy was performed on all the specimens to study and understand the effect of the gap between the microexciters and ITOF sensors on the sensor patch loadsensing performance. The distance between peak of a micro-exciter and ITOF sensors was recorded from each of the microscopic images. Analysis of the micrographs (Figure 12) suggests that specimens having better contact between ITOF sensors and microexciters are more likely to display better signal compared to specimens with the same configurations under the same loading condition but with no or less contact. Figure 12(a) shows no gap between micro-exciters and ITOF sensor, and the corresponding TL emission is shown in Figure 12( $\left.a^{\prime}\right)$. However, Figure 12(b) shows a large gap of $476 \mu \mathrm{m}$ between the peak of a microexciter and ITOF sensor, and the corresponding TL emission (low intensity) is shown in Figure 12( $\left.b^{\prime}\right)$. The gap between micro-exciters and ITOF sensor might be responsible for the large TL intensity variations. This result is expected because a large gap between the micro-exciters and ITOF sensor will result in a negligible amount of friction-induced signal, therefore a higher insensitivity to low load. It is now understood that to improve repeatability of signal generation, the fabrication process must address the variance in micro- 


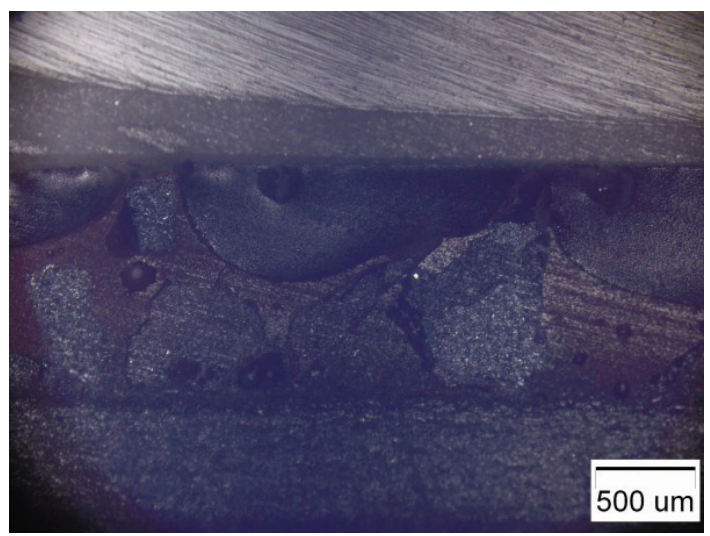

(a)

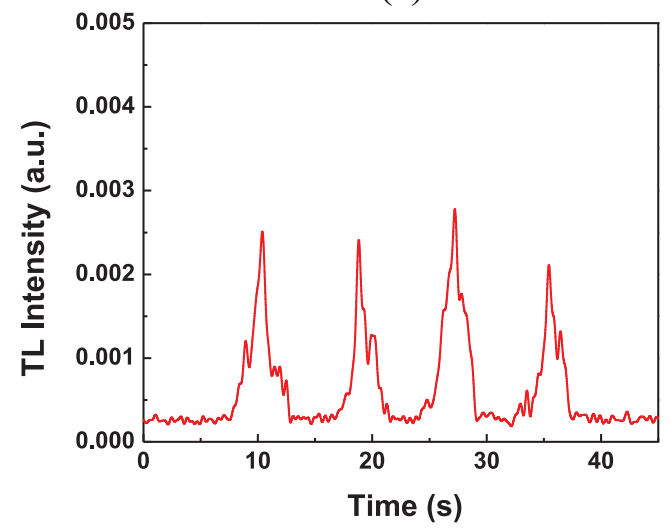

$\left(\mathrm{a}^{\prime}\right)$

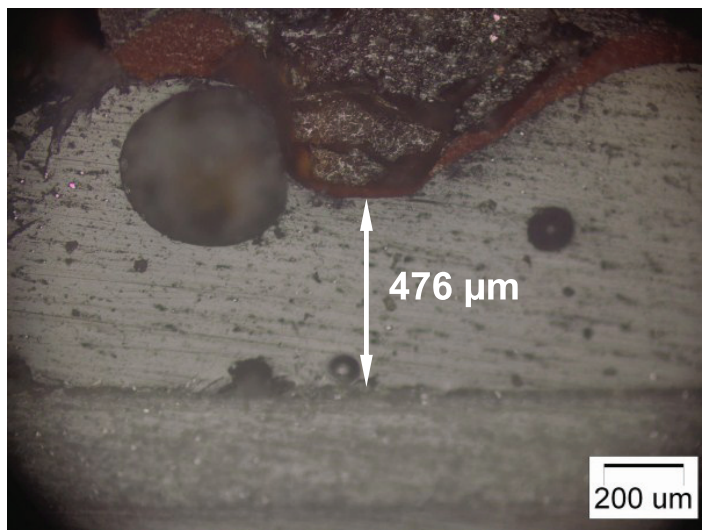

(b)

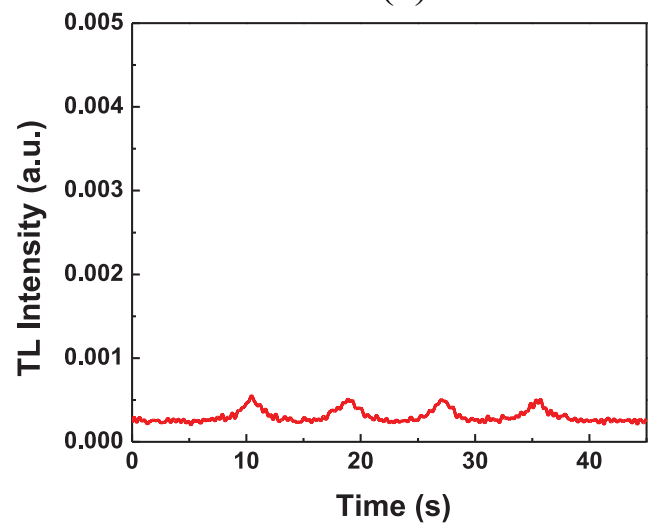

$\left(b^{\prime}\right)$

Figure 12. Optical micrographs of cross sections of specimens showing the interface between ITOF sensors and micro-exciters: (a) no gap between ITOF sensors and micro-exciters, ( $\left.\mathrm{a}^{\prime}\right)$ corresponding TL emissions, (b) polyurethane matrix layer (476 $\left.\mu \mathrm{m}\right)$ in between micro-exciters and ITOF sensors, and ( $\left.b^{\prime}\right)$ corresponding TL emissions.

exciter placement. Clearly, no gap between the sensor and micro-exciter is desirable so by altering the method of fabrication, a more repeatable sensor can be produced.

Scanning electron microscopy. SEM was also used to characterize the tested samples to gain further insights into the sensor network patch's structure and load-sensing performance. Four specimens among those used for the optical microscopy were studied with the SEM. Figure 13(a) shows a micrograph of a specimen where microexciters were in contact with the ITOF surface. The distance between micro-exciters and ITOF sensors was measured using ImageJ software from each of the microscopic images and plotted (for a 3.5-mm-long ITOF sensor) in Figure 13(b). The peak of the two micro-exciters touches the ITOF sensor. These specimens demonstrated higher TL intensity during testing. The micro-exciters excite the $\mathrm{ZnS}: \mathrm{Mn}$ crystals during loading as they lay on the ITOF sensors' surface.

Figure 14(a) shows a micrograph of specimen that did not show expected TL signals during loading. Figure 14(b) shows the distance between micro-exciters and ITOF sensors that was measured using ImageJ software from microscopic image (for 3.5-mm-long ITOF sensor). There is a polyurethane matrix layer in between micro-exciters and ITOF sensors. The range of polyurethane layer observed between the micro-exciters and the ITOF sensor is around $400-600 \mu \mathrm{m}$. This prevented the micro-exciters from exciting the TL crystals on the TL coating of the integrated ITOF sensors. As such, these samples did not produce the expected signal even though they had the same configuration as the sample shown in Figure 13.

\section{Conclusion}

This article has demonstrated a TL-based load monitoring sensor which can produce TL emissions corresponding to each loading cycle. Experimental results indicate that there is a clear correlation between TL emissions intensity values and the applied load on the ITOFPress. In general, the TL intensity increases exponentially with the increase in load and vice versa. Moreover, the higher the loading rate, the higher the TL intensity. Furthermore, the ITOFPress containing a 


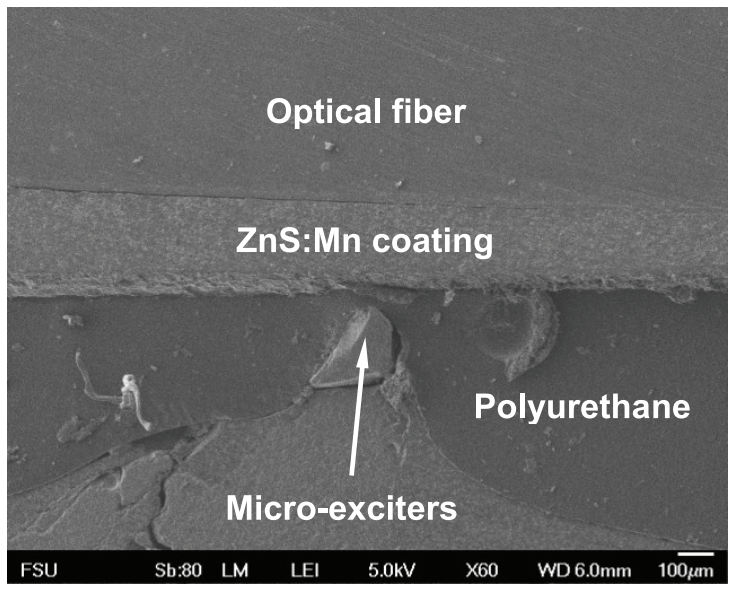

(a)

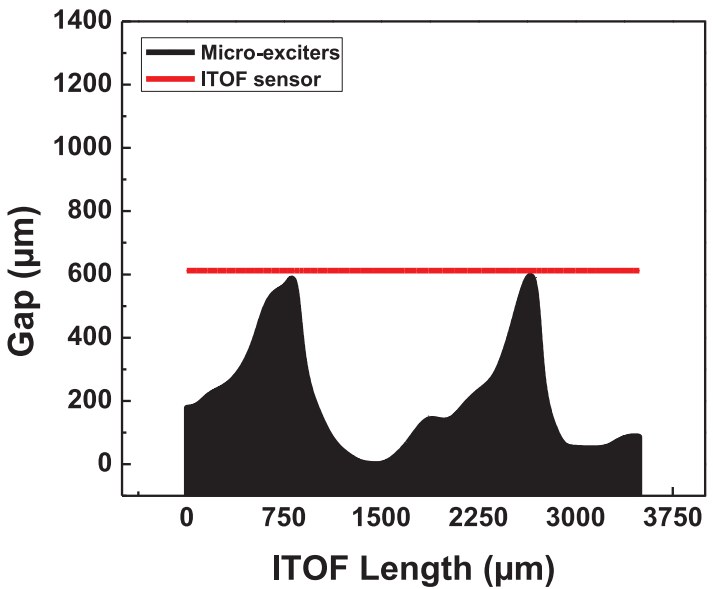

(b)

Figure 13. (a) SEM micrograph of sample with higher and consistent TL emission intensity shows the interface between ITOF sensors and micro-exciters (no gap). (b) The distance between micro-exciters and ITOF sensors from a typical microscopic image that shows two contacts with ITOF sensor. (The distance was measured by Imagej software.)

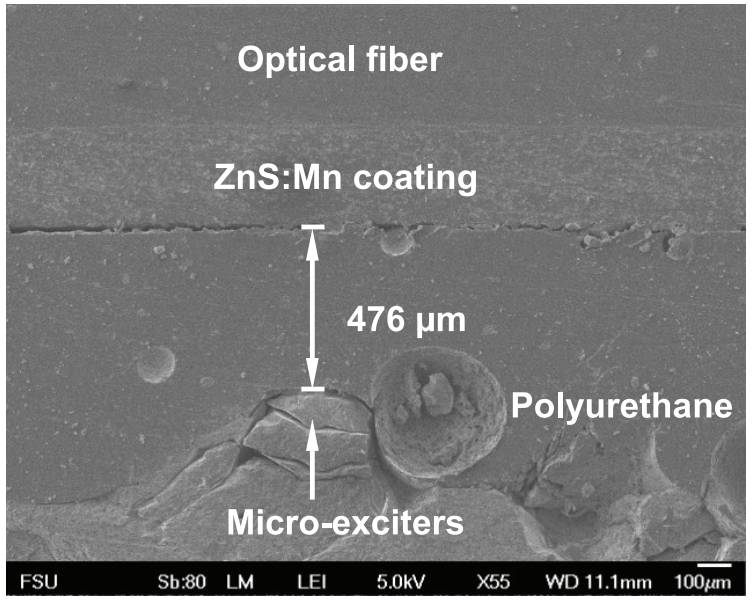

(a)

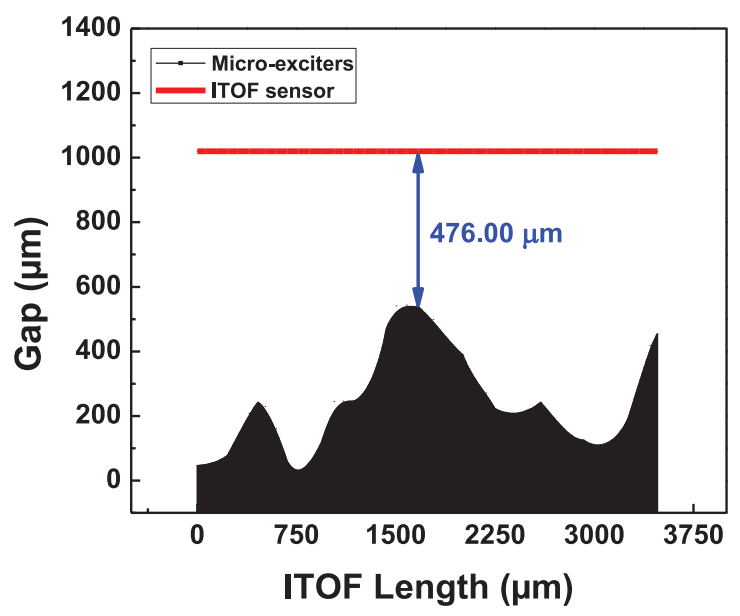

(b)

Figure 14. (a) SEM micrograph of sample with low or no TL emission intensity because of a 476- $\mu \mathrm{m}$ polyurethane layer between ITOF sensor and the peak of a micro-exciter. (b) The distance between micro-exciters and ITOF sensors from a typical microscopic image that shows two contacts with ITOF sensor. (The distance was measured by Imagej software.)

coarser micro-exciter (high coefficient of friction) exhibited better results. As such, it is concluded that the TL intensity $\left(I_{T L}\right)$ is a function of loading rate, coefficient of friction, and applied load on the sample.

The effect of patch matrix materials for improved load-sensing performance of the new ITOFPress was also investigated. The semi-flexible (matrix materialMaterial A) patch shows more consistent results for each cycle, but the TL intensity is comparatively low. On the other hand, for the stiffer patch (matrix material-Material B), the TL intensity was distinctly enhanced compared to the semi-flexible patch, although higher variation in signal intensity was observed. A higher TL intensity was observed during repeated loading due to the effective load transfer to ITOF sensors, but the load decreases significantly after each consecutive cycle. To obtain a consistent and high TL emission, a new patch matrix material as well as microexciters' host material are needed that can perform well under fatigue loading.

To correlate the TL intensity with the applied load, several loading and unloading half cycles were plotted for the two patch matrix materials and an empirical relationship is suggested as $I_{T L}=A \exp (F / B)+C_{0}$. The load sensitivity threshold is approximately $30 \%$ of the peak load for the sensor configuration used in this study. The work is underway to investigate the load sensitivity threshold to a minimum value and sensor 
long-term repeatability for better sensor performance. In addition, the work includes finding the optimum grit size of micro-exciters because there is a high possibility to rapidly wear out the $\mathrm{ZnS}: \mathrm{Mn}$ coating on ITOF sensor when using coarse micro-exciters. Additionally, during sensor fabrication, it is important to make sure that micro-exciters are in close proximity to the ITOF sensors, that is, no gap between them. Additional work is being done to optimize the sensor fabrication so that the sensor can be calibrated for load sensing. Fatigue tests will also be performed on the ITOF sensor patch to gain insight into its durability and performance under extended cyclic loading.

Clearly, to develop a fully functional load monitoring TL sensor, additional research would be required in the following areas:

1. Study of advanced materials such as patch matrix materials and micro-exciters' host materials, to eliminate the degradation trends as shown in Figure 11;

2. Data analysis of hundreds of cycles of loading to understand the long-term signal trend, sensor repeatability, and durability for sensor calibration;

3. Cyclic loading on ITOF sensor patch until an insignificant stress relaxation of patch matrix is obtained;

4. Developing a model based on experimental data to find out the correlations of TL intensity with applied loads and loading rates;

5. Optimization of sensor fabrication (fabrication consistency is another factor which will influence the sensor performance) and calibration.

Once fully developed, TL-based sensor patches will be able to provide real-time load monitoring on critical structures such as wind turbine blades for the active control of the turbine. Another potential application could be in high loading rate applications where conventional sensors might have difficulties.

\section{Declaration of conflicting interests}

The author(s) declared no potential conflicts of interest with respect to the research, authorship, and/or publication of this article.

\section{Funding}

The author(s) disclosed receipt of the following financial support for the research, authorship, and/or publication of this article: The authors wish to acknowledge the support of the National Science Foundation (NSF) under NSF Award No.: CMMI-0969413, NSF Award No. EEC-1005016; the Department of Energy under DOE Award No.: DE-
NA0000728; and the NSF and Nanotechnology Patronas Group Inc. (NPGroup) under Award No.: NSF-IIP 1549716.

\section{References}

Bergeron N, Hollerman W, Goedeke S, et al. (2006) Experimental evidence of triboluminescence induced by hypervelocity impact. International Journal of Impact Engineering 33: 91-99.

Chan TH, Yu L, Tam H-Y, et al. (2006) Fiber Bragg grating sensors for structural health monitoring of Tsing Ma bridge: background and experimental observation. Engineering Structures 28: 648-659.

Chandra VK and Chandra BP (2011) Suitable materials for elastico mechanoluminescence-based stress sensors. Optical Materials 34: 194-200.

Goedeke S, Allison S, Womack F, et al. (2003) Tribolumininescence and its application to space-based damage sensors. In: Proceedings of the propulsion measurement sensor development workshop, Huntsville, AL, 12-13 May.

Ho SCM, Ren L, Li H-N, et al. (2013) A fiber Bragg grating sensor for detection of liquid water in concrete structures. Smart Materials and Structures 22: 055012.

Hollerman WA, Fontenot RS, Bhat KN, et al. (2012) Comparison of triboluminescent emission yields for 27 luminescent materials. Optical Materials 34: 1517-1521.

Jia Y, Yei M and Jia W (2006) Stress-induced mechanoluminescence in $\mathrm{SrAl}_{2} \mathrm{O}_{4}: \mathrm{Eu}^{2+}, \mathrm{Dy}^{3+}$. Optical Materials 28: 974-979.

Leelachao S, Muraishi S, Sannomiya T, et al. (2016) Correlation of triboluminescence and contact stresses in $\mathrm{ZnS}: \mathrm{Mn} /$ polymeric matrix composite. Journal of Luminescence 170: 24-29.

Leng J, Barnes R, Hameed A, et al. (2006) Structural NDE of concrete structures using protected EFPI and FBG sensors. Sensors and Actuators A: Physical 126: 340-347.

Li D, Ho S-CM, Song G, et al. (2015) A review of damage detection methods for wind turbine blades. Smart Materials and Structures 24: 033001.

Majumder M, Gangopadhyay TK, Chakraborty AK, et al. (2008) Fibre Bragg gratings in structural health monitoring - present status and applications. Sensors and Actuators A: Physical 147: 150-164.

Moyo P, Brownjohn J, Suresh R, et al. (2005) Development of fiber Bragg grating sensors for monitoring civil infrastructure. Engineering Structures 27: 1828-1834.

Olawale DO (2013) In-situ triboluminescent optical fiber sensor for real-time damage monitoring in cementitious composites. PhD Thesis, Department of Industrial and Manufacturing Engineering, Florida State University, Tallahassee, Florida.

Olawale DO, Fontenot RS, Shohag MAS, et al. (2016a) Introduction to triboluminescence. In: Olawale DO, Okoli OI, Fontenot RS and et al. (eds) Triboluminescence: Theory, Synthesis, and Application. Berlin: Springer, pp. 1-16.

Olawale DO, Kliewer K, Okoye A, et al. (2014) Real time failure detection in unreinforced cementitious composites with triboluminescent sensor. Journal of Luminescence 147: 235-241.

Olawale DO, Sullivan W, Dickens TJ, et al. (2011) Mimicking the human nervous system with a triboluminescence sensory receptor for the structural health monitoring of 
composite structures. In: Proceedings of the SPIE 7981: sensors and smart structures technologies for civil, mechanical, and aerospace systems, San Diego, CA, 6 March.

Olawale DO, Uddin MJ, Yan J, et al. (2016b) Triboluminescent sensors for cement-based composites. In: Olawale DO, Okoli OI, Fontenot RS and et al. (eds) Triboluminescence: Theory, Synthesis, and Application. Berlin: Springer, pp. $379-410$.

Ozbek M, Rixen D and Verbruggen T (2009) Remote monitoring of wind turbine dynamics by laser interferometry: phase 1. In: Proceedings of the 27th international modal analysis conference, Orlando, FL, 9-12 February.

Qi HJ and Boyce MC (2005) Stress-strain behavior of thermoplastic polyurethanes. Mechanics of Materials 37: 817-839.

Sage I, Badcock R, Humberstone L, et al. (1999) Triboluminescent damage sensors. Smart Materials and Structures 8: 504.

Schroeder K, Ecke W, Apitz J, et al. (2006) A fibre Bragg grating sensor system monitors operational load in a wind turbine rotor blade. Measurement Science and Technology 17: 1167-1172.

Shohag MAS, Hammel EC, Dessureault Y-S, et al. (2016a) Triboluminescent sensor network for load monitoring in wind turbine blades. In: Proceedings of the CAMX conference, Anaheim, CA, 26-29 September. California, USA: CAMX, The Composites and Advanced Materials Expo, pp. 190-203. California, USA.

Shohag MAS, Hammel EC, Olawale DO, et al. (2016b) Adhesive bond failure monitoring with triboluminescent optical fiber sensor. In: Proceedings of the SPIE 9803: sensors and smart structures technologies for civil, mechanical, and aerospace systems, Las Vegas, NV, 20 March.

Someya S, Ishii K, Saeki M, et al. (2013) Lifetime-based measurement of stress using mechanoluminescence of $\mathrm{SrAl}_{2} \mathrm{O}_{4}: \mathrm{Eu}^{2+}$. Optics Letters 38: 1095-1097.

Sweeting LM (2001) Triboluminescence with and without air. Chemistry of Materials 13: 854-870.

Walton AJ (1977) Triboluminesence. Advances in Physics 26: 887-948.

Womack FN, Goedeke SM, Bergeron NP, et al. (2003) Measurement of triboluminescence and proton half brightness dose for ZnS:Mn. IEEE Transactions on Nuclear Science 51: 1737-1741.

Xu CN, Watanabe T, Akiyama M, et al. (1999a) Artificial skin to sense mechanical stress by visible light emission. Applied Physics Letters 74: 1236-1238.

Xu CN, Watanabe T, Akiyama M, et al. (1999b) Preparation and characteristics of highly triboluminescent $\mathrm{ZnS}$ film. Materials Research Bulletin 34: 1491-1500.

Yi J, Boyce MC, Lee GF, et al. (2006) Large deformation rate-dependent stress-strain behavior of polyurea and polyurethanes. Polymer 47: 319-329.

Zhang J-C, Xu C-N, Kamimura S, et al. (2013) An intense elastico-mechanoluminescence material CaZnOS:Mn2+ for sensing and imaging multiple mechanical stresses. Optics Express 21: 12976-12986. 